\section{A Model-Free Continuous Velocity Observer Formulation With Self- Tuning for Mechatronic Systems}

\author{
Meryem Deniz \\ Department of Electrical and Electronics Engineering, \\ Izmir Institute of Technology, \\ Urla 35430, Izmir, Turkey \\ e-mail: meryemdeniz@iyte.edu.tr
}

\author{
Alper Bayrak \\ Department of Electrical and Electronics Engineering, \\ Abant Izzet Baysal University, \\ Bolu 14280, Turkey \\ e-mail: alperbayrak@ibu.edu.tr

\section{Enver Tatlicioglu ${ }^{1}$} \\ Department of Electrical and Electronics Engineering, \\ Izmir Institute of Technology, \\ Urla 35430, Izmir, Turkey \\ e-mail: envertatlicioglu@iyte.edu.tr
}

\section{Erkan Zergeroglu}

Department of Computer Engineering,

Gebze Technical University,

Gebze 41400, Kocaeli, Turkey

e-mail: e.zerger@gtu.edu.tr

In this study, the design of a smooth robust velocity observer for a class of uncertain nonlinear mechatronic systems is presented. The proposed velocity observer does not require a priori knowledge of the upper bounds of the uncertain system dynamics and introduces time-varying observer gains for uncertainty compensation. Practical stability of the velocity observation error is ensured via Lyapunov-type stability analysis. Experimental results obtained from Phantom Omni haptic device are presented to illustrate the performance of the proposed velocity observer.

[DOI: $10.1115 / 1.4038373$ ]

\section{Introduction}

Most commercially available mechatronic systems including industrial robot manipulators are not equipped with a sensor for measuring velocities of the system. In the lack of velocity sensing, in the literature, the two common ways to obtain the velocity data are either by filtering the position data [1] or by designing an observer [2-4]. In filter-based approaches, usually a filter is designed to approximate the behavior of a differentiator over a range of frequencies which in most cases results in extra numerical noise insertion to the overall system. On the other hand, utilizing observers for velocity estimation as in Refs. [2-4] avoids the aforementioned drawback of filter-based techniques. While most of these velocity observers require the dynamical model of the mechatronic system to be partially or exactly known [5-7], there are robust observers $[3,4]$ that require only the upper bounds of the uncertain functions in the dynamical model. These robust observers can roughly be grouped as the ones that utilize signum function in their designs (i.e., sliding mode observers or their

\section{Corresponding author}

Contributed by the Dynamic Systems Division of ASME for publication in the Journal of Dynamic Systems, Measurement, and Control. Manuscript received June 3, 2016; final manuscript received October 10, 2017; published online December 19, 2017. Assoc. Editor: Azim Eskandarian. modifications) $[3,4,8-15]$, and the ones that require higher observer gains (i.e., high-gain observers) [16-18]. While the discontinuous nature of the signum function is considered to be useful in the stability analysis, the researchers seeked for alternatives to replace them especially for some implementations where smoothness is required (such as telerobotic applications). Another important issue in designing nonlinear observers is the gain tuning, which is usually not a straightforward task. In most robust observers in the literature, a priori knowledge of the bounds of the dynamic modeling functions are required for gain tuning.

In response to the above design questions, a novel continuous velocity observer for nonlinear mechatronic systems is aimed. In a seemingly novel departure from the existing results in the literature, in this paper, hyperbolic tangent function is utilized in the observer design and its associated analysis is presented. The use of hyperbolic tangent function ensures continuity of the velocity observer when compared with the variable structure observers. The proposed observer design includes time-varying observer gains to avoid the need of a priori knowledge of the upper bounds of the uncertain mechatronic system dynamics. This is the main novelty of the proposed velocity observer. Lyapunov-type stability analysis techniques are utilized to demonstrate practical tracking. The performance of the proposed observer is demonstrated by experiments performed on Phantom Omni haptic device.

\section{Dynamic Model and Properties}

The following general form of mechatronic system dynamic model is considered in this work:

$$
\ddot{x}=h+G u
$$

where $x(t) \in \mathbb{R}^{n}$ is the position with $\dot{x}(t), \ddot{x}(t) \in \mathbb{R}^{n}$ being velocity and acceleration of the mechatronic system, respectively, $u(t) \in \mathbb{R}^{n}$ is the control input, $h(x, \dot{x}, t) \in \mathbb{R}^{n}$ and $G(x, \dot{x}, t) \in$ $\mathbb{R}^{n \times n}$ are uncertain nonlinear functions of position and velocity of the mechatronic system, respectively. The position vector $x(t)$ is the output of the mechatronic system which is denoted by $y(t) \in$ $\mathbb{R}^{n}$ (i.e., $y=x$ ).

Remark 1. It is noted that the $n$ degree-of-freedom robot manipulator model $M(\theta) \ddot{\theta}+V_{m}(\theta, \dot{\theta}) \dot{\theta}+F_{d}(\dot{\theta})+G(\theta)+\tau_{d}(t)=\tau(t)$ with $x \triangleq \theta, G \triangleq M^{-1}, u \triangleq \tau$ and $h \triangleq-M^{-1}\left(V_{m} \dot{\theta}+F_{d}+G+\tau_{d}\right)$ can be represented as in the general mechatronic system model in Eq. (1).

The subsequent observer development requires the dynamic model in Eq. (1) to satisfy the following standard assumptions:

Assumption 1. The nonlinear functions are $h(x, \dot{x}), G(x, \dot{x})$, and the control input $u(t)$ are $\mathcal{C}^{1}$. The control input and its time derivative are bounded functions of time (i.e., $\left.u(t), \dot{u}(t) \in \mathcal{L}_{\infty}\right)$. The system output $x(t)$ and its time derivative $\dot{x}(t)$ remain bounded for all time (i.e., $x(t), \dot{x}(t) \in \mathcal{L}_{\infty}$ ).

\section{Observer Design}

The objective is to design a continuous observer to estimate the velocity of a mechatronic system. A velocity observer signal, denoted by $\dot{\hat{x}}(t) \in \mathbb{R}^{n}$, will be designed to observe $\dot{x}(t)$ while ensuring the velocity observation error, defined as $\dot{\tilde{x}}(t) \triangleq \dot{x}(t)$ $-\dot{\hat{x}}(t)$, approach to a finite and acceptable small region around the origin. The design problem is restricted by the constraint that the right-hand side of the system model in Eq. (1) being uncertain thus the observer design should be model free. In addition, we want to design update rules for the gains of the velocity observer thus the observer gains will be auto-tuned.

To achieve these objective, based on the subsequent stability analysis, the velocity observer is designed as

$$
\dot{\hat{x}}=p+\left(K+I_{n}\right) \tilde{x}
$$

where $K(t) \in \mathbb{R}^{n \times n}$ is a positive definite, diagonal, time-varying observer gain matrix, $I_{n} \in \mathbb{R}^{n \times n}$ is the standard identity 
matrix, and $\tilde{x}(t) \in \mathbb{R}^{n}$ is the position observation error defined as

$$
\tilde{x} \triangleq x-\hat{x}
$$

The term $p(t) \in \mathbb{R}^{n}$ in Eq. (2) denotes an auxiliary signal updated according to

$$
\dot{p}=K \tilde{x}+\hat{\beta} \tanh (\tilde{x})
$$

where $\hat{\beta}(t) \in \mathbb{R}^{n \times n}$ is a positive definite, diagonal, time-varying observer gain matrix and $\tanh (\tilde{x}) \in \mathbb{R}^{n}$ is the vector form of the hyperbolic tangent function defined as

$$
\tanh (\tilde{x}) \triangleq\left[\tanh \left(\tilde{x}_{1}\right), \ldots, \tanh \left(\tilde{x}_{n}\right)\right]^{\mathrm{T}}
$$

for $\tilde{x} \triangleq\left[\tilde{x}_{1}, \ldots, \tilde{x}_{n}\right]^{\mathrm{T}}$. The entries of the time-varying observer gain matrices $K(t)$ and $\hat{\beta}(t)$ are updated according to

$$
\begin{gathered}
K_{i}(t)=k_{c}+\frac{1}{2} \tilde{x}_{i}^{2}(t)-\frac{1}{2} \tilde{x}_{i}^{2}(0)+\int_{0}^{t} \tilde{x}_{i}^{2}(\sigma) d \sigma \\
\hat{\beta}_{i}(t)=\ln \left(\cosh \left(\tilde{x}_{i}(t)\right)\right)+\int_{0}^{t} \tilde{x}_{i}(\sigma) \tanh \left(\tilde{x}_{i}(\sigma)\right) d \sigma
\end{gathered}
$$

where subscript $i$ denotes the entries of a vector or a diagonal matrix which is $\forall i=1, \ldots, n$ with $k_{c}$ being a positive constant.

Remark 2 . The velocity observer in Ref. [15] is given as

$$
\dot{\hat{x}}=p+\left(K_{2}+I_{n}\right) \tilde{x} \quad \text { with } \dot{p}=K_{1} \operatorname{sgn}(\tilde{x})+K_{2} \tilde{x}
$$

where $K_{1}$ and $K_{2}$ are constant observation gain matrices and $\operatorname{sgn}(\cdot)$ is the vector signum function. The main difference between the velocity observer in this paper and the velocity observer in Ref. [15] is that, as opposed to the constant observer gain matrices $K_{1}$ and $K_{2}$ of Ref. [15], we designed time-varying observer gains (i.e., $K(t)$ in Eq. (6) and $\hat{\beta}(t)$ in Eq. (7)). From Eq. (52) of Ref. [15], it is required that the entries of the matrix $K_{1}$ be selected larger than the upper bounds of model uncertainties which is not the case for the proposed time-varying gains. Another important difference of the proposed velocity observer when compared to that of Ref. [15] is that we used hyperbolic tangent function as opposed to the signum function.

To ease the presentation of subsequent Lyapunov analysis, an auxiliary error signal, denoted by $r(t) \in \mathbb{R}^{n}$, is introduced as follows:

$$
r \triangleq \dot{\tilde{x}}+\tilde{x}
$$

Taking the time derivative of Eq. (2) and substituting Eq. (4) yields

$$
\ddot{\hat{x}}=K r+\dot{\tilde{x}}+\hat{\beta} \tanh (\tilde{x})+\dot{K} \tilde{x}
$$

where Eq. (8) was also utilized. Substituting Eqs. (1) and (9) into the time derivative of Eq. (8) results in

$$
\dot{r}=-K r+N-\hat{\beta} \tanh (\tilde{x})-\dot{K} \tilde{x}
$$

where the auxiliary signal $N(t) \in \mathbb{R}^{n}$ is defined as

$$
N \triangleq h+G u
$$

It is highlighted that, in view of assumption 1, it is clear that $N(t)$ and $\dot{N}(t)$ are bounded functions of time.

\section{Stability Analysis}

Lyapunov-based methods will be utilized in investigating the stability of the proposed observer. In the design of the Lyapunov function, in addition to quadratic terms, an auxiliary function will be utilized. The auxiliary function $V_{L}(t) \in \mathbb{R}$ is defined as

$$
V_{L} \triangleq \zeta_{L}-L
$$

where $L(t) \in \mathbb{R}$ is defined as

$$
L \triangleq \int_{0}^{t} r^{\mathrm{T}}(\sigma)[N(\sigma)-\beta \tanh (\tilde{x}(\sigma))] d \sigma
$$

with $\beta \in \mathbb{R}^{n \times n}$ being a positive definite diagonal constant matrix, and $\zeta_{L} \in \mathbb{R}$ is a positive constant defined as

$$
\zeta_{L} \triangleq \sum_{i=1}^{n} \beta_{i}\left(\ln \left[\cosh \left(\tilde{x}_{i}(0)\right)\right]+1\right)-\tilde{x}^{\mathrm{T}}(0) N(0)
$$

Lemma 1. When $\tilde{x}(t)$ is outside of a hyperball around the origin denoted by $d(\varepsilon)$ with $\varepsilon$ being a positive design parameter specifying the radius of the hyperball, the auxiliary function $V_{L}(t)$ can be lower bounded in the sense that

$$
V_{L}(t) \geq 0
$$

provided that the entries of $\beta$ satisfy

$$
\beta_{i}>\frac{1}{\varepsilon}\left(\left\|N_{i}\right\|_{\infty}+\left\|\dot{N}_{i}\right\|_{\infty}\right)
$$

where the notation $\|\cdot\|_{\infty}$ denotes the supremum of a time-varying signal.

Proof. The proof of this Lemma is similar to the one in Ref. [19].

Remark 3. As can be seen from the proof of Lemma 1 in Ref. [19], if $\varepsilon=0$, then, it would be possible to drive the velocity observer error to zero. But as $\varepsilon \rightarrow 0$ then, from Eq. (16), it is clear that $\beta \rightarrow+\infty$. As this would constitute a problem, from Eqs. (2)-(7), $\beta$ is not utilized in the observer design and instead the time-varying observer gain $\hat{\beta}(t)$ was introduced and utilized.

THEOREM 1. The velocity observer design in Eqs. (2)-(7) ensures ultimate asymptotic convergence of velocity observation error $\dot{\tilde{x}}(t)$ to a hyperball around the origin with an adjustable radius.

Proof. The following novel Lyapunov function, denoted by $V(t) \in \mathbb{R}$, is introduced:

$$
V \triangleq V_{L}+\frac{1}{2} r^{\mathrm{T}} r+\frac{1}{2} \sum_{i=1}^{n} \tilde{\beta}_{i}^{2}
$$

where

$$
\tilde{\beta}_{i} \triangleq \beta_{i}-\hat{\beta}_{i}
$$

and $V_{L}(t)$ was previously defined in Eq. (12). Note that the expression in Eq. (17) is positive and lower bounded provided that $V_{L}(t) \geq 0$ (i.e., when $\|\tilde{x}\| \geq d(\varepsilon)$ from Lemma 1) and thus on the same region satisfies

$$
\frac{1}{2}\|w\|^{2} \leq V(t, w) \leq\|w\|^{2} \quad \forall\|w\| \geq\|\tilde{x}\| \geq d(\varepsilon)
$$

where $w \triangleq\left[\begin{array}{lllll}r^{\mathrm{T}} & \tilde{\beta}_{1} & \ldots & \tilde{\beta}_{n} & \sqrt{V_{L}}\end{array}\right]^{\mathrm{T}} \in \mathbb{R}^{(2 n+1) \times 1}$.

Taking the time derivative of Eq. (17), and utilizing the time derivative of $V_{L}(t)$ in Eq. (12) along with the time derivative of $L(t)$ in Eq. (13), and substituting Eq. (10) and time derivative of Eq. (6), we obtain

$$
\begin{aligned}
\dot{V}= & r^{\mathrm{T}}(-N+\beta \tanh (\tilde{x})) \\
& +r^{\mathrm{T}}(-K r+N-\hat{\beta} \tanh (\tilde{x}))-\sum_{i=1}^{n} r_{i}^{2} \tilde{x}_{i}^{2}-\sum_{i=1}^{n} \tilde{\beta}_{i} \dot{\hat{\beta}}_{i}
\end{aligned}
$$



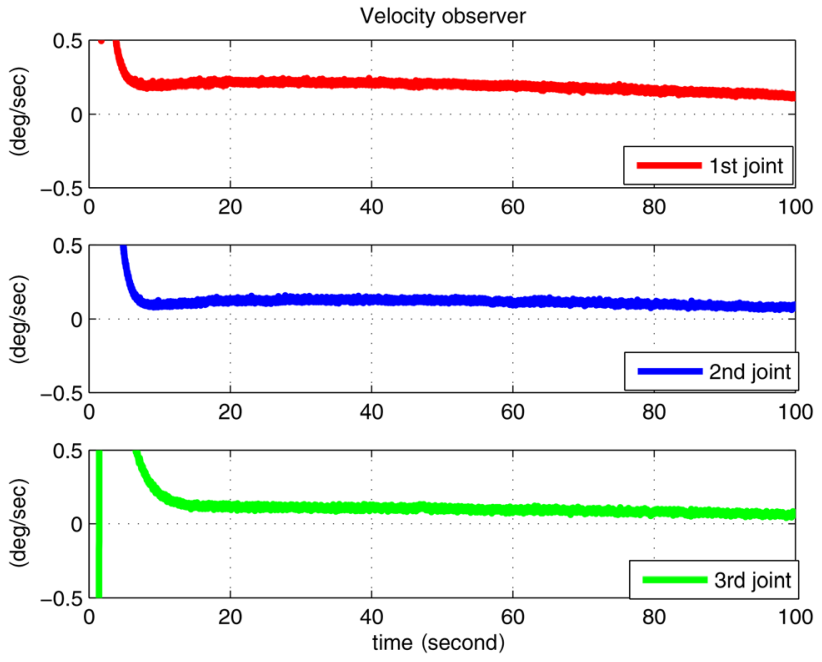

Fig. 1 Velocity observer $\dot{\hat{x}}(t)$

where the time derivative of Eq. (18) was also utilized. After utilizing the time derivative of Eq. (7) along with Eq. (20), the following expression is obtained:

$$
\dot{V}=r^{\mathrm{T}}(\beta-\hat{\beta}) \tanh (\tilde{x})-r^{\mathrm{T}} K r-\sum_{i=1}^{n} r_{i}^{2} \tilde{x}_{i}^{2}-\sum_{i=1}^{n} \tilde{\beta}_{i} r_{i} \tanh \left(\tilde{x}_{i}\right)
$$

and after considering the fact

$$
r^{\mathrm{T}}(\beta-\hat{\beta}) \tanh (\tilde{x})=\sum_{i=1}^{n} r_{i} \tilde{\beta}_{i} \tanh \left(\tilde{x}_{i}\right)
$$

the following upper bound is reached:

$$
\dot{V} \leq-k_{c} \geq\|r\|^{2} \quad \forall\|w\| \geq\|\tilde{x}\| \geq d(\varepsilon)
$$

From the structures of Eqs. (17) and (23), it is clear that $V(t) \in$ $\mathcal{L}_{\infty}$ and thus $r(t), V_{L}(t), \tilde{\beta}(t) \in \mathcal{L}_{\infty}$. From the definition of $r(t)$, observation errors $\tilde{x}(t), \dot{\tilde{x}}(t) \in \mathcal{L}_{\infty}$ [20]. From the structures of Eqs. (17) and (23), it can be stated that the observation errors will approach into the hyperball shown with $d(\varepsilon)$ as time increases provided that Eq. (16) is satisfied.
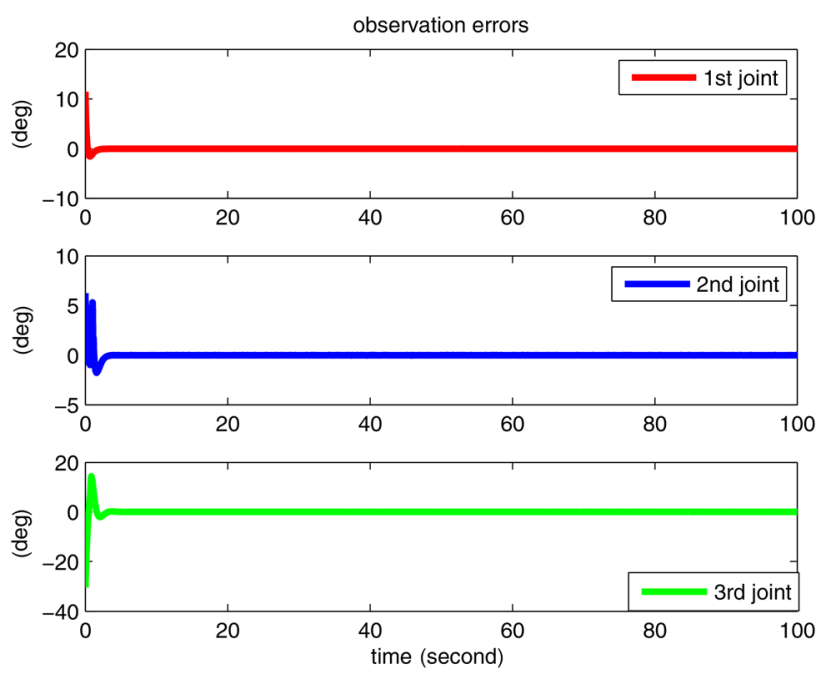

Fig. 2 Position observation error $\tilde{\boldsymbol{x}}(t)$
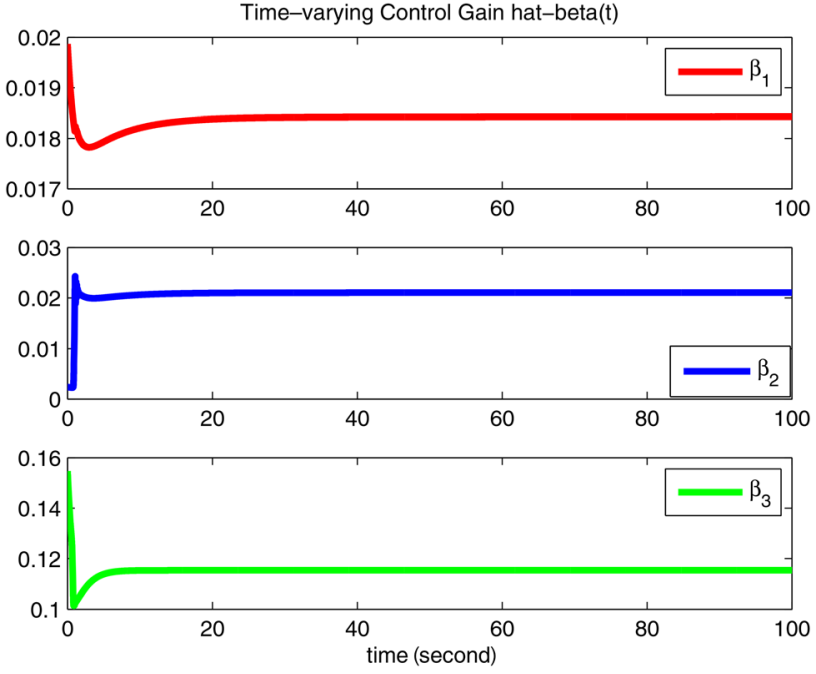

Fig. 3 Time-varying observer gain $\hat{\beta}(t)$

Remark 4. While the convergence proof of the velocity observer formulation proposed in this work is inspired from the analysis presented in Ref. [19], we would like to emphasize that Dasdemir and Zergeroglu [19] did not propose an observer formulation but a high-gain controller with constant control gains. In this work, a velocity observer with time-varying gains has been designed. We have extended the controller design presented in Ref. [19] by applying it to an observer design and fusing a timevarying gain formulation. We would also want to point out that the aforementioned task is not straightforward as the corresponding design with the stability analysis requires significant rework and modifications.

\section{Experiment Results}

In this section, the performance of the proposed velocity observer is demonstrated by experiment results obtained from Phantom Omni haptic device. The experimental studies run on MATLAB SIMULINK with a data rate of $100 \mathrm{~Hz}$. Time-varying observer gain includes a positive constant gain which was chosen as $0.4 \times \operatorname{diag}([0.12 ; 0.2 ; 0.2])$. The initial position of Phantom omni haptic device was chosen as $[0 ; 0.26 ; 0.5]^{\mathrm{T}}$ radians, and $\hat{x}(0)$ was chosen as $[-0.2 ; 0.16 ; 0.16]^{\mathrm{T}}$ radians.

The results of experimental studies are shown in Figs. 1-4. Figure 1 represents the observed velocities $\dot{\hat{x}}(t)$ for each joint. In
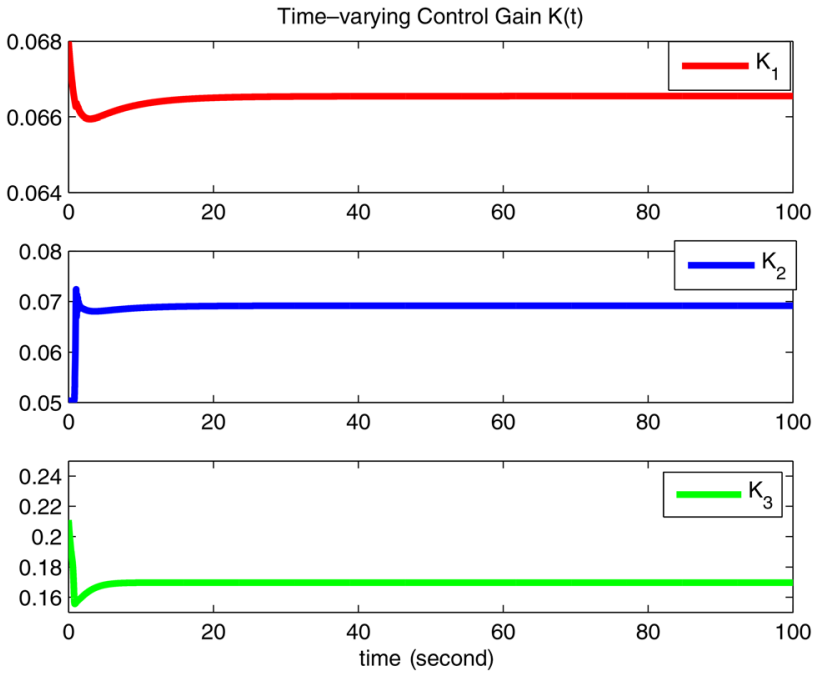

Fig. 4 Time-varying observer gain $K(t)$ 
Fig. 2, the position observation error $\tilde{x}(t)$ is presented. In Figs. 3 and 4, time-varying observer gains $\hat{\beta}(t)$ and $K(t)$ are presented. From Fig. 2, it is seen that the position observation error is driven to the vicinity of zero and thus the objective is achieved.

\section{Conclusions}

In this work, a new model-free continuous velocity observer was designed for a class of uncertain nonlinear mechatronic systems. The smoothness of the velocity observer was ensured via the use of hyperbolic tangent functions in the design. The design of the velocity observer differs from the similar observer techniques in the literature in the sense that the proposed observer utilizes time-varying observer gains with only one scalar constant gain (i.e., $k_{c}$ in Eq. (6)) which being positive suffices. Ultimate asymptotic stability of the observer error was ensured via Lyapunov-type stability analysis. Experimental results performed on a robot manipulator were presented to illustrate the performance of the proposed observer.

\section{Funding Data}

- The Scientific and Technological Research Council of Turkey (Grant No. 113E147).

\section{References}

[1] Su, Y. X., Zheng, C. H., Mueller, P. C., and Duan, B. Y., 2006, “A Simple Improved Velocity Estimation for Low-Speed Regions Based on Position Measurements Only," IEEE Trans. Control Syst. Technol., 14(5), pp. 937-942.

[2] Arimoto, S., Parra-Vega, V., and Naniwa, T., 1994, "A Class of Linear Velocity Observers for Nonlinear Mechanical Systems," Asian Control Conference, Tokyo, Japan, July 27-30, pp. 633-636.

[3] Abdessameud, A., and Khelfi, M. F., 2006, "A Variable Structure Observer for the Control of Robot Manipulators," Int. J. Appl. Math. Compt. Sci., 16(2), pp. 189-196.
[4] de Wit, C. C., and Slotine, J., 1991, "Sliding Observers for Robot Manipulators," Automatica, 27(5), pp. 859-864.

[5] Astolfi, A., Ortega, R., and Venkatraman, A., 2010, "A Globally Exponentially Convergent Immersion and Invariance Speed Observer for Mechanical Systems With Non-Holonomic Constraints," Automatica, 46(5), pp. 182-189.

[6] Namvar, M., 2009, "A Class of Globally Convergent Velocity Observers for Robotic Manipulators,” IEEE Trans. Autom. Control, 54(8), pp. 1956-1961.

[7] Romero, J. G., and Ortega, R., 2015, "Two Globally Convergent Adaptive Speed Observers for Mechanical Systems," Automatica, 60, pp. 7-11.

[8] Choi, J.-H., Misawa, E. A., and Young, G. E., 1999, “A Study on Sliding Mode State Estimation,” ASME J. Dyn. Syst. Meas. Control, 121(6), pp. 255-260.

[9] Davila, J., Fridman, L., and Levant, A., 2005, "Second-Order Sliding-Mode Observer for Mechanical Systems," IEEE Trans. Autom. Control, 50(11), pp. 1785-1789.

[10] Dawson, D. M., Qu, Z., and Carroll, J. C., 1992, "On the State Observation and Output Feedback Problems for Nonlinear Uncertain Dynamic Systems," Syst. Control Lett., 18(2), pp. 217-222.

[11] González, I., Salazar, S., and Lozano, R., 2014, "Chattering-Free Sliding Mode Altitude Control for a Quad-Rotor Aircraft: Real-Time Application,” J. Intell. Rob. Syst., 73(1-4), pp. 137-155.

[12] Ramirez-Rodriguez, H., Parra-Vega, V., Sanchez-Orta, A., and Garcia-Salazar, O., 2014, "Robust Backstepping Control Based on Integral Sliding Modes for Tracking of Quadrotors," J. Intell. Rob. Syst., 73(1-4), pp. 51-66.

[13] Walcott, B., and Zak, S., 1987, "State Observation of Nonlinear Uncertain Dynamical Systems," IEEE Trans. Autom. Control, 32(2), pp. 166-170.

[14] Xiong, Y., and Saif, M., 2001, "Sliding Mode Observer for Nonlinear Uncertain Systems," IEEE Trans. Autom. Control, 46(12), pp. 2012-2017.

[15] Xian, B., de Queiroz, M., Dawson, D., and McIntyre, M., 2004, “A Discontinuous Output Feedback Controller and Velocity Observer for Nonlinear Mechanical Systems," Automatica, 40(4), pp. 695-700.

[16] Atassi, A. N., and Khalil, H. K., 1999, "A Separation Principle for the Stabilization of a Class of Nonlinear Systems," IEEE Trans. Autom. Control, 44(9), pp. 1672-1687.

[17] Chen, J., Behal, A., and Dawson, D., 2008, "Robust Feedback Control for a Class of Uncertain MIMO Nonlinear Systems," IEEE Trans. Autom. Control, 53(2), pp. 591-596.

[18] Teel, A., and Praly, L., 1994, "Global Stabilizability and Observability Imply Semi Global Stabilizability by Output Feedback," Syst. Control Lett., 22(2), pp. 313-325.

[19] Dasdemir, J., and Zergeroglu, E., 2015, "A New Continuous High-Gain Controller Scheme for a Class of Uncertain Nonlinear Systems," Int. J. Robust Nonlinear Control, 25(1), pp. 125-141.

[20] Lewis, F., Dawson, D., and Abdallah, C., 2004, Robot Manipulator Control: Theory and Practice, Marcel Dekker, New York. 